\title{
PVST, a tool to assess the Power to Volume scaling distortions associated to code simulations
}

\author{
V. Martínez-Quiroga ${ }^{\mathrm{a}, *}$, J. Freixa ${ }^{\mathrm{a}}$, F. Reventós ${ }^{\mathrm{a}}$ \\ ${ }^{a}$ Department of Physics and Nuclear Engineering, Technical University of Catalonia (UPC), Spain
}

\begin{abstract}
System codes along with necessary nodalizations are valuable tools for thermal hydraulic safety analysis. In order to assess the safety of a particular power plant, in addition to the validation and verification of the code, the nodalization of the system needs to be qualified. Since most existing experimental data come from tests performed on scaled-down facilities, any qualification process must therefore address scale considerations. Along these lines, the Group of Thermal Hydraulic Studies at Technical University of Catalonia (GET) developed SCUP, a scaling-up methodology for qualifying full-scale nodalizations through a systematic procedure based on the extrapolation of post-test simulations of Integral Test Facilities (ITF) experiments. For that purpose, GET created the "Power-to-Volume-Scaling Tool" (PVST), a RELAP5mod3 add-on software to easily generate scaled-up input decks following the Power to Volume (PtoV) methodology. The present paper describes the main features of this software as well as the scaling distortions that can be expected from applying PtoV to the RELAP5mod3 equations. In this sense, a detailled description of the PtoV methodology is introduced together with the particular criteria applied in the design of the main components of an ITF. In addition, an exhaustive study of the RELAP5mod3 equations is carried out to identify which are dimensionally affected by the PtoV scaling factors. Finally, PVST scaling analyses capabilities are assessed on two post-test experiments that were carried out at the Large Scale Test Facility (LSTF) within the framework of the OECD/NEA ROSA and ROSA-2 projects. Results demonstrated the high reliability of the software for identifying those particular phenomena (environment heat losses, broken legs flow regime transitions, subcooled liquid vapour mixing, ...) that modify the experiment simulations because of the PtoV scaling criterion.
\end{abstract}

Keywords: PWR, scaling issue, RELAP5mod3, Power to Volume scaling

\section{Introduction}

"Scaling" in system thermal-hydraulics applications has been and still is a key word in the field of nuclear reactor safety and design. Since the 1970s, the concerns on scaling have been focused on both the design of experimental facilities and the validity of thermal-hydraulic system codes regardless of the scale of the problem being addressed. A recent OECD initiative has led to an updating debate in the involved scientific community in which a certain consensus has been reached on the general picture of the issue. The words "scaling issue" indicate the complexity and difficulty of the process. The initiative results are collected in OECD/NEA (2017), in which commonly-accepted topics and the controversial ones are described in detail. Following such update, the current work plan at the Advanced Nuclear Technologies (ANT) of the Universitat Politecnica de Catalunya (UPC) includes different developments dealing with the consolidation of scaling issue own practices. The most relevant development is a scaling-up methodology (SCUP) for

\footnotetext{
*Corresponding author. Tel.: +34-934017153.

Email addresses: victor.martinez.quiroga@upc.edu (V. Martínez-Quiroga), jordi.freixa-terrades@upc.edu (J. Freixa), francesc.reventos@upc.edu (F. Reventós)
} 
qualifying full-scale nodalizations by the use of the Design and Beyond Design Basis Accident (DBA and BDBA) experiments performed at Integral Test Facilities (ITF) of reduced scale.

ITFs are designed with the aim of analyzing the whole response of a commercial NPP under accidental conditions. Emergency Operational Procedures (EOPs), Accident Management and safety margins are the targets of their design and therefore it is essential to reproduce thermal-hydraulic phenomena as close as possible to their reference plant. Most of the operative ITFs in the world were designed according to the Power-to-Volume (PtoV) scaling principle. This approach is based on the studies of Navahandi et al. (1979), who demonstrated the scalability of the balance equations for single phase 1-dimensional vertical flows. The method intends to conserve time and heat flux in the experimental facility by reducing both the power and the volume with the same scaling value.

The SCUP methodology, extensively described in Martinez-Quiroga and Reventos (2014b) and Freixa and et al. (2016), establishes a systematic procedure for estimating the effects of the ITF configuration and its scaling down criterion to the system codes simulations of those scenarios. For that purpose, a specific scaling tool, PVST, was designed. This program is an add-on to RELAP5, a thermal-hydraulic system code developed by the Idaho National Engineering Laboratory (INEL) for the U.S. Nuclear Regulatory Commission. The PVST tool "duplicates" RELAP5mod3 input decks at different scales by applying the scaling factors of the Power to Volume Scaling method. The present publication addresses the capabilities and usage of the PVST tool in the frame of the PtoV scaling analysis. The following sections address the limitations and distortions implied by the PtoV method in the design process of ITFs; and the scalability of the RELAP5 code results by using this method. These two steps are required to understand the purpose, capabilities and application of the PVST tool which is described afterwards. In order to enhance their comprehension, further details of PtoV method as well as RELAP5mod3 system equations are included in this section.

\subsection{Power to Volume scaling method}

Power to volume scaling is one of the most common methods used for the design of ITF, especially for those Pressurized Water Reactor (PWR) experimental facilities built during the 80s and 90s. PtoV scaling criteria are obtained by simplifying the balance equations of the system under certain assumptions and requirements that are related with the inherent characteristics of Light Water Reactors (LWR) Ybarrondo and et al. (1974), Cabernier and Chudnik (1969), Navahandi et al. (1979). Hence, taking into account that reactor flows are mainly dominated by large sources and sinks of power and momentum (core power, high depressurization rates, pumps, ...), it is considered that interphase interactions can be omitted (equal velocity and temperature for both liquid and vapor phases) and that mass, momentum and energy equations can be analyzed as one directional single phase vertical fluid (radial fluxes are also neglected). Thus, for such conditions one can write the balance equations of mass, energy and momentum in non dimensional form as follows

$$
\begin{gathered}
\frac{\partial \rho^{+}}{\partial t^{+}}+\left[\frac{v_{0} t_{0}}{l_{0}}\right] \frac{\partial \rho^{+} v^{+}}{\partial x^{+}}=0 \\
\rho^{+} \frac{\partial v^{+}}{\partial t^{+}}+\left[\frac{v_{0} t_{0}}{l_{0}}\right] \rho^{+} v^{+} \frac{\partial v^{+}}{\partial x^{+}}=-\left[\frac{P_{0}}{l_{0}} \frac{t_{0}}{\rho_{0} v_{0}}\right] \frac{\partial P^{+}}{\partial x^{+}}-\left[\frac{\xi_{0}}{A_{c 0}} K v_{0} t_{0}\right] \frac{\xi^{+} \rho \kappa v^{2}+}{A_{c}^{+}}-\left[\frac{g t_{0}}{v_{0}}\right] g^{+} \rho^{+} \\
\rho^{+} \frac{\partial h^{+}}{\partial t^{+}}+\left[\frac{v_{0} t_{0}}{l_{0}}\right] \rho^{+} v^{+} \frac{\partial h^{+}}{\partial x^{+}}=\left[\frac{\xi_{0}}{A_{c 0}} \frac{q_{0} t_{0}}{\rho_{0} h_{0}}\right] \frac{\xi^{+} q^{+}}{A_{c}^{+}}-\left[\frac{P_{0}}{\rho_{0} h_{0}}\right] \frac{\partial P^{+}}{\partial t^{+}}-\left[\frac{v_{0} t_{0}}{l_{0}} \frac{P_{0}}{\rho_{0} h_{0}}\right] v^{+} \frac{\partial P^{+}}{\partial x^{+}}
\end{gathered}
$$

Where superscript + makes reference to normalized parameters and subscript zero to the normalizing values of the chosen (reference) plant.

For obtaining the power to volume scaling factors, it is considered that equations (1) to (3) must be equal for both the reference plant and the ITF. This implies the following equalities must be fulfilled: 


$$
\begin{aligned}
{\left[\frac{v t}{l}\right]_{R} } & =\left[\frac{v t}{l}\right]_{I T F} \\
{\left[\frac{P}{\rho} \frac{t}{l v}\right]_{R} } & =\left[\frac{P}{\rho} \frac{t}{l v}\right]_{I T F} \\
{\left[\frac{\xi}{A_{c}} K v t\right]_{R} } & =\left[\frac{\xi}{A_{c}} K v t\right]_{I T F} \\
{\left[\frac{g t}{v}\right]_{m} } & =\left[\frac{g t}{v}\right]_{I T F} \\
{\left[\frac{\xi}{A_{c}} \frac{q t}{\rho h}\right]_{R} } & =\left[\frac{\xi}{A_{c}} \frac{q t}{\rho h}\right]_{I T F} \\
{\left[\frac{P}{\rho h}\right]_{R} } & =\left[\frac{P}{\rho h}\right]_{I T F}
\end{aligned}
$$

In order to preserve the equalities of (4), the power-to-volume approach requires that the fluid properties and pressure are the same in both systems, and that lengths in the direction of the fluid are preserved, thus the following correspondences are valid:

$$
\frac{P_{R}}{P_{I T F}}=\frac{\rho_{R}}{\rho_{I T F}}=\frac{h_{R}}{h_{I T F}}=\frac{l_{R}}{l_{I T F}}=\frac{t_{R}}{t_{I T F}}=1
$$

With these assumptions, the following power-to-volume relationships are obtained:

$$
\begin{gathered}
\frac{\phi_{R}}{\phi_{I T F}}=\frac{W_{R}}{W_{I T F}}=\frac{V_{R}}{V_{I T F}}=\frac{A_{R}}{A_{I T F}}=K_{v} \\
{\left[\frac{\xi}{A_{c}} K\right]_{R}=\left[\frac{\xi}{A_{c}} K\right]_{I T F}}
\end{gathered}
$$

Full details o the derivation of the PtoV scaling factors were given by Zuber (1980). It is worth mentioning that condition (7) cannot be accomplished since perimeter $(\xi)$ depends on the diameters of the component to be scaled and they change in the scaling process by applying the $K_{v}$ factor to the cross sectional area of the fluid (7). Hence, given that power to volume scaling conditions cannot be perfectly preserved between the facility and the reference plant, and that some assumptions, like the omissions of the interphase interactions in the balance equations, are decisive for the proper reproduction of the thermalhydraulic processes in specific components(e.g. heat exchange in the core and the SGs), a particular definition of the scaling factors is needed and carried out depending on the components of the LWR to be scaled in the ITF. A further description about how is specifically applied the power to volume scaling methodology in the design of the experimental facilities can be found in Section 2.

\subsection{RELAP5mod3}

RELAP5mod3 is a light water reactor transient analysis code framed within best estimates codes (like CATHARE, TRAC, ATHLET...), which try to reproduce realistically the physical phenomena in thermalhydraulics systems without considering conservative assumptions. This kind of codes started to become relevant after Three Mile Island (1979), time in which industry and national regulatory bodies considered necessary to develop complex software that analyzes a wider variety of postulated accident conditions minimizing the amount of physical assumptions on the part of the user.The code is designed to simulate any component of a LWR system, as well as nuclear power generation, heat transfer processes between fluid and metal structures, and the whole plant control system and boundary conditions.

About RELAP5mod3 physics, several approximations and assumptions are performed in the system equations in order to simulate conveniently the thermalhydraulics of the fluid, the heat conduction inside 
the metal structures, and the transport of non-condensables and non volatile solute (boron) for the particular LWR systems.

The RELAP5mod3 thermalhydraulic model is based on a nonhomogeneous and nonequilibrium model, which simulates a two-phase fluid system plus transport of non-condensables and non volatile solute (boron). For the fluid system, balance equations (mass, momentum and energy) are simplified: on the one hand, only one dimension is considered and vapour and liquid are decoupled, thus independent equations for both phases are obtained. On the other hand, since nuclear reactor flows are dominated by large sources and sinks of momentum, viscous stresses and other secondary momentum effects are considered secondary to mass and energy conservation for reactor safety analysis, and momentum equations are reduced to Bernoullis equations for steady, incompressible and frictionless flow. Finally, differential equations are discretized for the numerical solution applying time and space averaging. As a result of all these approximations, effects like turbulent fluctuations, axial diffusion and transversal gradients and fluxes, which affect decisively to interfacial and wall interaction processes, are neglected. Hence, it becomes necessary to introduce flow regime maps and constitutive equations to simulate the fluid behavior realistically. These constitutive models compute those effects independently in specific correlations. Their results, that vary depending on the kind of flow regime, are added to the final solution of the field equations by the terms like FWG, FWF, DISS, FI, $\Gamma, \mathrm{C}$ and Q. Furthermore, extra correlations have been also added to RELAP5mod3 in order to simulate properly special processes that are not computed by the state equations (choked flow, momentum losses due to abrupt area changes, ...).

Regarding heat conduction process, heat structures included in RELAP5mod3 permit the calculation of the heat transferred across solid boundaries of hydrodynamic volumes. They are represented by one dimensional heat conduction equations in rectangular, cylindrical, or spherical geometries, which are discretized in the radial direction. In addition, a two-dimensional heat conduction model has been included in order to simulate axial heat conduction during core rewetting processes. For this model, both rectangular and cylindrical coordinates can be used, with reference to a $2 \mathrm{D}$ node.

\section{ITF design: Application of the Power to Volume Scaling Methodology}

Integral Test Facilities are designed with the aim of analyzing the whole response of a commercial NPP under accidental conditions. EOPs, Accident Management and safety margins are the targets of their design, hence it becomes essential to reproduce thermalhydraulic phenonema as close as possible to their reference plant. As shown in the introduction, several simplifications are applied in the PtoV scaling approach, thus specific transcription of the scaling factors is necessary depending on the component and the relevance of the thermalhydraulic processes to reproduce. In the following subsections it is considered that coolant is the same for both the ITF and the reference plant, and hence, fluid properties conditions of relationship (5) are accomplished.

\subsection{Vertical volumes}

Vertical volumes are scaled-down in order to preserve the inventory ratio, the balance equations under operational conditions (liquid single phase) and the pressures drops along the flow path. Thus, ITF and reference plant heights are the same in order to keep water columns, and volumes are scaled with the $K_{v}$ factor just modifying the cross sectional area:

$$
\begin{gathered}
(\rho g H)_{R}=(\rho g H)_{I T F} \\
\frac{A_{R}}{A_{I T F}}=K_{v}
\end{gathered}
$$

Components like vessel and SGs plenums are scaled-down following these relationships. On the other hand, downcomers of SGs and reactor pressure vessel (RPV) can show relevant mixture conditions for accident scenario and require a particular expert judgment for the scaling-down: subcooled water mixes with saturated coolant when Auxiliary Feed Water (AFW) and Emergency Core Coolant Systems (ECCSs) 


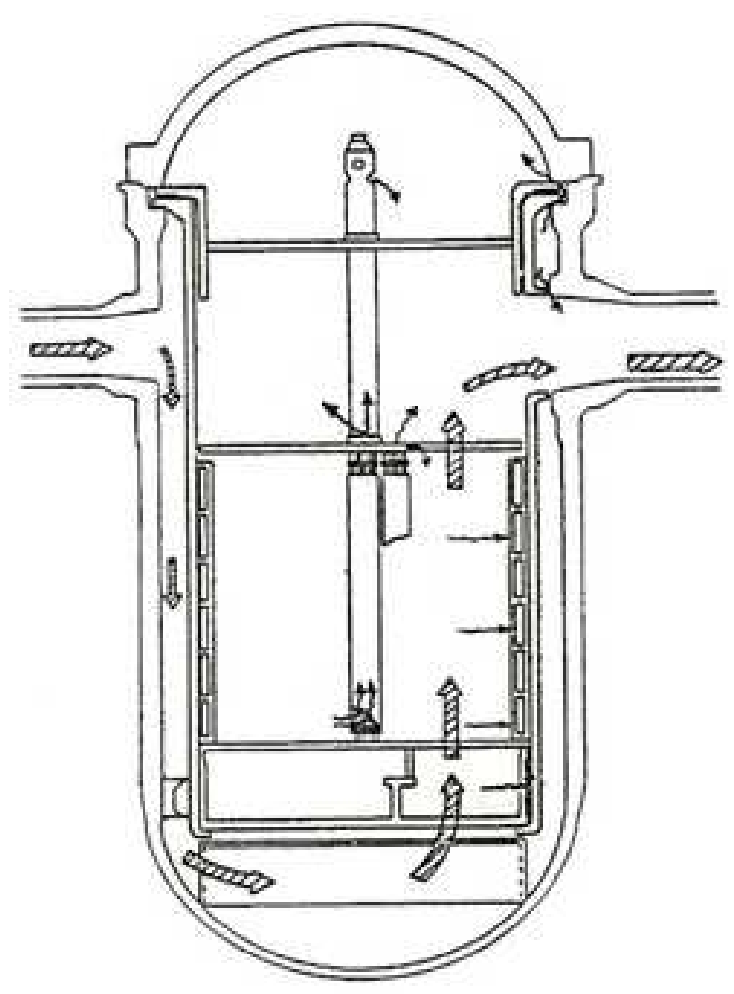

typical PWR

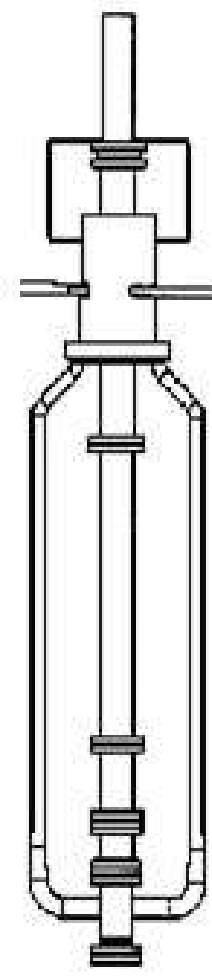

ITF

Figure 1: Vertical volumes in reactor vessel Kremin et al. (2001)

are operative. If it occurs under shut-down conditions (low power), interphase frictions, mass and heat transfers cannot be neglected and they can affect to the correct reproduction of the reactor cooldown. Thus, in some ITFs designs, downcomers are scaled-down following (8) and (9), but also modifying the hydraulic diameter in order to minimize the impossibility to reach (7) and the impact of the single phase assumption in the power to volume scaling approach. In any case, despite the engineering approaches, it is impossible to remove completely the effect of the hydraulic diameter in the friction losses and the interphase interactions, thus scaling distortions cannot be completely avoided under these particular accident conditions. In Figure 1 it is shown a comparison between the vessel design of a NPP and PKL, a test facility of AREVA Kremin et al. (2001). It can be noticed that downcomer has been modified from an annulus to two pipes so that the diameters are modified but the heights and scaled-down volumes are preserved. With this approach the hydraulic diameter is increased and becomes close to that of the downcomer in the reference plant.

\subsection{Multi-channel regions}

For multi-channel regions in which the relevant heat exchange processes of the facility take place, i.e. core region and SG riser, it is essential to scale identical flow regimes and heat transfers behavior independently of the transient conditions. Thus, since the PtoV scaling approach neglects two-phase interactions, a multichannel criterion is applied in order to preserve identical geometries and flow paths. This consists of keeping the geometries of the NPP channels (heights, hydraulic diameters and pitch-to-rod diameter ratio) but reducing their number by the $K_{v}$ factor for the ITF and thus preserving the volume scaling criterion: 


$$
\begin{aligned}
V_{R}=V_{\text {chan }} \cdot\left(\text { num.chan }_{R}\right) & =K_{v} V_{\text {chan }} \cdot\left(\text { num.chan }_{I T F}\right)=K_{v} V_{I T F} \\
D_{R} & =D_{I T F} \\
H_{R} & =H_{I T F}
\end{aligned}
$$

With this criterion, as flow paths are identical, thermalhydraulic processes are identical in each channel (without considering radial distribution feedback) and power to volume scaling factors are preserved for an overall application of the scaling approach.

\subsection{Horizontal legs}

Flow regime transitions in horizontal volumes (cold and hot legs) become essential during SBLOCA transients. Depending on their coolant mixture conditions, break discharging, core quenching, and primaryto-secondary heat transfer will be affected. Thus, for a proper scaling-down of horizontal legs, it is necessary to preserve flow regime transitions.

Therefore, an additional power to volume scaling relationship is considered based on classical works performed by Taitel and Dukler (1976) Taitel et al. (1980). They observed, as it is shown in Figure 2, that flow regime transitions depends on the horizontal stratified level and the Froude number which must be preserved. Hence, for preserving Froude number as well as the power to volume scaling conditions for the volume and the mass flow rate

$$
\begin{aligned}
\left(\frac{v_{g \sqrt{\rho_{g}}}}{\sqrt{g \Delta \rho D}}\right)_{R} & =\left(\frac{v_{g \sqrt{\rho_{g}}}}{\sqrt{g \Delta \rho D}}\right)_{I T F} \\
W_{R} & =K_{v} W_{I T F} \\
V_{R} & =K_{v} V_{I T F}
\end{aligned}
$$

it must be accomplished that

$$
\left(\frac{L}{\sqrt{D}}\right)_{R}=\left(\frac{L}{\sqrt{D}}\right)_{I T F}
$$

On the other hand, as diameters and lenghts are not preserved, (7) is not accomplished and scaling distortions can be expected at very low pressures because of the frictional effects.

\subsection{Environment heat losses}

Another important aspect to be considered in the design of an ITF is the effect of the energy that is lost to the environment. In particular, if PtoV scaling method is applied, environment heat losses do not change with the same $K_{v}$ factor as volumes and power (6). For vertical volumes, power transferred by conduction and convection processes changes as

$$
\begin{gathered}
\phi_{w R}=-k_{R} A_{R} \frac{\partial T_{R}}{\partial x_{R}}=-k_{I T F} \pi D_{R} L_{R} \frac{\partial T_{I T F}}{\partial x_{I T F}} \\
=-\sqrt{K_{v}} k_{I T F} A_{I T F} \frac{\partial T_{I T F}}{\partial x_{I T F}}=\sqrt{K_{v}} \phi_{w I T F} \\
\phi_{w R}=-h_{c R} A_{R}\left(T_{w}-T_{f}\right)=-h_{c I T F} \pi D_{R} L_{R}\left(T_{w}-T_{f}\right) \\
=-\sqrt{K_{v}}-h_{c I T F} A_{I T F}\left(T_{w}-T_{f}\right)=\sqrt{K_{v}} \phi_{w I T F}
\end{gathered}
$$

and for horizontal volumes, 


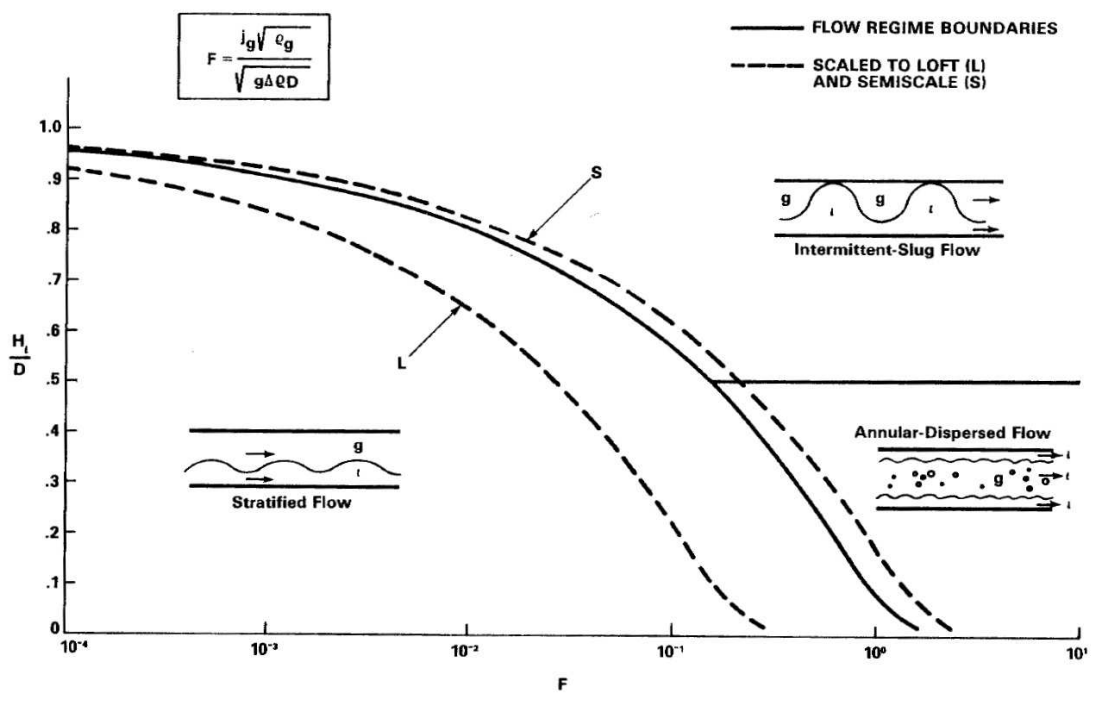

Figure 2: Dukler-Taitel flow regime map Taitel and Dukler (1976)

$$
\begin{gathered}
\phi_{w R}=-k_{R} A_{R} \frac{\partial T_{R}}{\partial x_{R}}=-k_{I T F} \pi\left(K_{v}\right)^{2 / 5} D_{I T F}\left(K_{v}\right)^{1 / 5} L_{I T F} \frac{\partial T_{I T F}}{\partial x_{I T F}} \\
=-\left(K_{v}\right)^{3 / 5} k_{I T F} A_{I T F} \frac{\partial T_{I T F}}{\partial x_{I T F}}=\left(K_{v}\right)^{3 / 5} \phi_{w I T F} \\
\phi_{w R}=-h_{c R} A_{R}\left(T_{w}-T_{f}\right)=-h_{c I T F} \pi\left(K_{v}\right)^{2 / 5} D_{I T F}\left(K_{v}\right)^{1 / 5} L_{I T F}\left(T_{w}-T_{f}\right) \\
=-\left(K_{v}\right)^{3 / 5} h_{c I T F} A_{I T F}\left(T_{w}-T_{f}\right)=\left(K_{v}\right)^{3 / 5} \phi_{w I T F}
\end{gathered}
$$

Hence, the impact of the environment heat losses is not preserved in the ITF and scaling distortions are expected. For reducing the environment heat losses, the walls of the ITF pipes are usually covered by thick rockwool insulators and heaters may be used in several parts of the facility to compensate for heat losses.

\section{RELAP5mod3: expected Power to volume Scaling distortions}

The comparison between prototype RELAP5mod3 calculations and its related PtoV scaled-up calculations will yield inevitably scaling distortions. As it is described in relationship (5), scaling factors are obtained by considering that pressures, fluid properties and time are preserved. Hence, if RELAP5mod3 input decks are scaled, fluid tables and initial conditions (P,U, $\alpha$ and $\rho$ ) will be kept equal. On the contrary, geometries and volumetric parameters of the components will be modified depending on the $K_{v}$ factors (see equation (6)). As a result of these considerations, some differences will exist in the particular solutions of the RELAP5mod3 equations of both the prototype and the scaled-up input models. In the Phd Thesis Martinez-Quiroga (2014), there is an extensive description of the complete set of RELAP5mod3 equations and correlations (The RELAP5 Code Development Team (2001a) and The RELAP5 Code Development Team $(2001 b)$ ) as well as their dependencies with the geometrical parameters of the RELAP5mod3 components. A summary of the expected differences when applying the Power to Volume scaling factors are listed below depending on the type of component. 


\subsection{Vertical components}

For vertical components, volume and area are scaled with the same $K_{v}$ factor, thus, initial velocities and cell lenghts (height) are equal in both nodalizations by accomplishing the relationships (8) and 5 of the PtoV scaling method applied to vertical components. Considering Relap5mod3 balance equations ((17) to (22)), identical expressions are obtained after replacing known parameters (initial values, geometries and time step), hence scaling distortions can only exist if the terms of the closure equations and special processes are different $\left(\Gamma_{g}, Q_{i, g}, Q_{i, f}, Q_{w, g}, Q_{w, f}\right.$ DISS, FWF, FWG, FI, HLOSSG, HLOSSF, $\left.C_{M}\right)$. As it is shown in Sections 4.3.1.3.2, 4.3.1.3.3 and 4.3.1.3.5 of Martinez-Quiroga (2014), interphase interactions, wall friction and wall heat exchange depend on the hydraulic diameter and the flow regime, which in turn depends on the hydraulic diameter as well. Therefore, scaling distortions can be expected for vertical components under mixture conditions as a result of differences in hydraulic diameter.

$$
\begin{aligned}
& \left(\alpha_{g} \rho_{g}+\alpha_{f} \rho_{f}\right)_{j}^{n+1}-\left(\alpha_{g} \rho_{g}+\alpha_{f} \rho_{f}\right)_{j}^{n}+\left\{\left[\dot{\alpha}_{g}^{n} \dot{\rho}_{g}^{n}\left(v_{g}\right)_{j+1 / 2}^{n+1}+\dot{\alpha}_{f}^{n} \dot{\rho}_{f}^{n}\left(v_{f}\right)_{j+1 / 2}^{n+1}\right] A_{j+1 / 2}\right. \\
& \left.-\left[\dot{\alpha}_{g}^{n} \dot{\rho}_{g}^{n}\left(v_{g}\right)_{j-1 / 2}^{n+1}+\dot{\alpha}_{f}^{n} \dot{\rho}_{f}^{n}\left(v_{f}\right)_{j-1 / 2}^{n+1}\right] A_{j-1 / 2}\right\}\left(\Delta t / V_{j}\right)=0 \\
& \left(\alpha_{g} \rho_{g}-\alpha_{f} \rho_{f}\right)_{j}^{n+1}-\left(\alpha_{g} \rho_{g}-\alpha_{f} \rho_{f}\right)_{j}^{n}+\left\{\left[\dot{\alpha}_{g}^{n} \dot{\rho}_{g}^{n}\left(v_{g}\right)_{j+1 / 2}^{n+1}-\dot{\alpha}_{f}^{n} \dot{\rho}_{f}^{n}\left(v_{f}\right)_{j+1 / 2}^{n+1}\right] A_{j+1 / 2}\right. \\
& \left.-\left[\dot{\alpha}_{g}^{n} \dot{\rho}_{g}^{n}\left(v_{g}\right)_{j-1 / 2}^{n+1}-\dot{\alpha}_{f}^{n} \dot{\rho}_{f}^{n}\left(v_{f}\right)_{j-1 / 2}^{n+1}\right] A_{j-1 / 2}\right\}\left(\Delta t / V_{j}\right)=2\left(\Gamma_{g}\right)_{j}^{n+1} \Delta t \\
& \left(\alpha_{g} \rho_{g} U_{g}\right)_{j}^{n+1}-\left(\alpha_{g} \rho_{g} U_{g}\right)_{j}^{n}+P_{j}^{n}\left(\alpha_{g}^{n+1}-\alpha_{g}^{n}\right) \\
& +\left[\left(\dot{\alpha}_{g} \dot{\rho}_{g} \dot{U}_{g}\right)_{j+1 / 2}^{n}+\left(\dot{\alpha}_{g}^{n}\right)_{j+1 / 2} P_{j}^{n}\right]\left(v_{g}\right)_{j+1 / 2}^{n+1} A_{j+1 / 2}\left(\Delta t / V_{j}\right) \\
& -\left[\left(\dot{\alpha}_{g} \dot{\rho}_{g} \dot{U}_{g}\right)_{j-1 / 2}^{n}+\left(\dot{\alpha}_{g}^{n}\right)_{j-1 / 2} P_{j}^{n}\right]\left(v_{g}\right)_{j-1 / 2}^{n+1} A_{j-1 / 2}\left(\Delta t / V_{j}\right) \\
& =\left\{\left[-h_{f}^{*} /\left(h_{g}^{*}-h_{f}^{*}\right)\right]^{n} Q_{i g}^{n+1}-\left[h_{g}^{*} /\left(h_{g}^{*}-h_{f}^{*}\right)\right]^{n} Q_{i g}^{n+1}+Q_{w g}^{n}+D I S S_{g}^{n}\right\}_{j} \Delta t \\
& \left(\alpha_{f} \rho_{f} U_{f}\right)_{j}^{n+1}-\left(\alpha_{f} \rho_{f} U_{f}\right)_{j}^{n}+P_{j}^{n}\left(\alpha_{f}^{n+1}-\alpha_{f}^{n}\right) \\
& +\left[\left(\dot{\alpha}_{f} \dot{\rho}_{f} \dot{U}_{f}\right)_{j+1 / 2}^{n}+\left(\dot{\alpha}_{f}^{n}\right)_{j+1 / 2} P_{j}^{n}\right]\left(v_{f}\right)_{j+1 / 2}^{n+1} A_{j+1 / 2}\left(\Delta t / V_{j}\right) \\
& -\left[\left(\dot{\alpha}_{f} \dot{\rho}_{f} \dot{U}_{f}\right)_{j-1 / 2}^{n}+\left(\dot{\alpha}_{f}^{n}\right)_{j-1 / 2} P_{j}^{n}\right]\left(v_{f}\right)_{j-1 / 2}^{n+1} A_{j-1 / 2}\left(\Delta t / V_{j}\right) \\
& =\left\{\left[-h_{f}^{*} /\left(h_{g}^{*}-h_{f}^{*}\right)\right]^{n} Q_{i g}^{n+1}-\left[h_{g}^{*} /\left(h_{g}^{*}-h_{f}^{*}\right)\right]^{n} Q_{i g}^{n+1}+Q_{w f}^{n}+D I S S_{f}^{n}\right\}_{j} \Delta t
\end{aligned}
$$




$$
\begin{aligned}
& {\left[\left(\alpha_{g} \rho_{g}\right)_{j+1 / 2}^{n}\left(v_{g}^{n+1}-v_{g}^{n}\right)_{j+1 / 2}+\left(\alpha_{f} \rho_{f}\right)_{j+1 / 2}^{n}\left(v_{f}^{n+1}-v_{f}^{n}\right)_{j+1 / 2}\right] \Delta x_{j+1 / 2}} \\
& +1 / 2\left(\alpha_{g} \rho_{g}\right)_{j+1 / 2}^{n}\left[\left(v_{g}^{2}\right)_{j+1}^{n}-\left(v_{g}^{2}\right)_{j}^{n}\right] \Delta t+1 / 2\left(\alpha_{f} \rho_{f}\right)_{j+1 / 2}^{n}\left[\left(v_{f}^{2}\right)_{j+1}^{n}-\left(v_{f}^{2}\right)_{j}^{n}\right] \Delta t \\
& -1 / 2\left[\left(\alpha_{g} \rho_{g}\right)_{j+1 / 2}^{n} V I S G_{j+1 / 2}^{n}+\left(\alpha_{f} \rho_{f}\right)_{j+1 / 2}^{n} V I S F_{j+1 / 2}^{n}\right] \Delta t=-\left(P_{j+1}-P_{j}\right)^{n+1} \Delta t \\
& +\left[\rho_{j+1 / 2}^{n} g-\left(\alpha_{g} \rho_{g}\right)_{j+1 / 2}^{n}\left(v_{g}\right)_{j+1 / 2}^{n+1} F W G_{j+1 / 2}^{n}-\left(\alpha_{f} \rho_{f}\right)_{j+1 / 2}^{n}\left(v_{f}\right)_{j+1 / 2}^{n+1} F W F_{j+1 / 2}^{n}\right. \\
& \left.-\left(\Gamma_{g}\right)_{j+1 / 2}^{n}\left(v_{g}-v_{f}\right)_{j+1 / 2}^{n}\right] \Delta x_{j+1 / 2} \Delta t+\left[\left(\dot{\alpha}_{g} \dot{\rho}_{g}\right)_{j+1 / 2}^{n} H L O S S G_{j+1 / 2}^{n} v_{g j+1 / 2}^{n+1}\right. \\
& \left.+\left(\dot{\alpha_{f}} \dot{\rho_{f}}\right)_{j+1 / 2}^{n} \operatorname{HLOSSF_{j+1/2}^{n}} v_{f j+1 / 2}^{n+1}\right] \\
& \left(1+\frac{C_{M} \rho_{m}^{2}}{\rho_{g} \rho_{f}}\right)_{j+1 / 2}^{n}\left[\left(v_{g}^{n+1}-v_{g}^{n}\right)-\left(v_{f}^{n+1}-v_{f}^{n}\right)\right]_{j+1 / 2} \Delta x_{j+1 / 2} \\
& +\frac{1}{2}\left(\frac{\dot{\alpha_{g}} \dot{\rho}_{g}}{\alpha_{g} \rho_{g}}\right)_{j+1 / 2}^{n}\left[\left(v_{g}^{2}\right)_{j+1}^{n}-\left(v_{g}^{2}\right)_{j}^{n}\right] \Delta t-\frac{1}{2}\left(\frac{\dot{\alpha}_{g} \dot{\rho}_{g}}{\alpha_{g} \rho_{g}}\right)_{j+1 / 2}^{n} V I S G_{j+1 / 2}^{n} \Delta t \\
& +\frac{1}{2}\left(\frac{\dot{\alpha_{f}} \dot{\rho_{f}}}{\alpha_{f} \rho_{f}}\right)_{j+1 / 2}^{n}\left[\left(v_{f}^{2}\right)_{j+1}^{n}-\left(v_{f}^{2}\right)_{j}^{n}\right] \Delta t-\frac{1}{2}\left(\frac{\dot{\alpha_{f}} \dot{\rho}_{f}}{\alpha_{f} \rho_{f}}\right)_{j+1 / 2}^{n} V I S F_{j+1 / 2}^{n} \Delta t \\
& =-\left(\frac{\rho_{f}-\rho_{g}}{\rho_{f} \rho_{g}}\right)_{j+1 / 2}^{n}\left(P_{j+1}-P_{j}\right)^{n+1} \Delta t \\
& -\left\{F W G_{j+1 / 2}^{n}\left(v_{g}\right)_{j+1 / 2}^{n+1}-F W F_{j+1 / 2}^{n}\left(v_{f}\right)_{j+1 / 2}^{n+1}-\left[\frac{\Gamma_{g}^{n}\left(\rho_{m}^{n} v_{I}^{n+1}-\alpha_{f}^{n} \rho_{f}^{n} v^{n+1}-\alpha_{g}^{n} \rho_{g}^{n} v^{n+1}\right)}{\left(\alpha_{g} \rho_{g} \alpha_{f} \rho_{f}\right)^{n}}\right]_{j+1 / 2}\right. \\
& \left.+\left(\rho_{m} F I\right)_{j+1 / 2}^{n}\left(v_{g}^{n+1}-v_{f}^{n+1}\right)_{j+1 / 2}\right\} \Delta x_{j+1 / 2} \Delta t-\left[\frac{1}{2}\left(\frac{\dot{\alpha}_{g} \dot{\rho}_{g}}{\alpha_{g} \rho_{g}}\right)_{j+1 / 2}^{n} H L O S S G_{j+1 / 2}^{n} v_{g, j+1 / 2}^{n+1}\right. \\
& \left.-\frac{1}{2}\left(\frac{\dot{\alpha}_{f} \dot{\rho_{f}}}{\alpha_{f} \rho_{f}}\right)_{j+1 / 2}^{n} H \operatorname{LOSSF} F_{j+1 / 2}^{n} v_{f, j+1 / 2}^{n+1}\right] \Delta t+\left(\frac{\rho_{m}}{\rho_{g} \rho_{f}}\right)_{j+1 / 2}^{n}\left(\rho_{f}-\rho_{g}\right)_{j+1 / 2}^{n} g\left(H_{j+1}^{n}-H_{j}^{n}\right) \Delta t
\end{aligned}
$$

Other special processes like mixture level tracking model and thermal stratification model do not add scaling distortion given that elevations are preserved and equations (23), (24), (25), (26), (27) do not change after applying the scaling criteria.

$$
\begin{gathered}
d z l_{L}=d z_{L}\left(\frac{U_{f L}^{+}-U_{f L}}{U_{f L}^{+}-U_{f L}}\right) \\
v_{f r o n t}=\frac{d z l_{L}^{n}-d z l_{L}^{n-1}}{\Delta t} \\
H_{i}^{n e q} f=H_{i f}\left(1-\frac{d z l_{L}}{d z L}\right) \\
q_{w g}=h_{g}\left(T_{w}-T_{g}\right)\left(1-\frac{d z l_{L}}{d z_{L}}\right) \\
q_{w f}=h_{f}\left(T_{w}-T_{f}\right)\left(\frac{d z l_{L}}{d z_{L}}\right)
\end{gathered}
$$




\subsection{Multi-channel regions}

No scaling distortions are associated to multi-channel regions given that geometries, fluid properties and initial conditions are identical for both nodalizations (see scaling factor in section 2.2). Anyhow, if crossflows are defined between multi-channels (i.e. parallel channels in a PWR core nodalization), scaling distortions can be noticed under asymmetrical transient conditions (i.e. main steam line break). This behavior occurs because radial lengths are not preserved $\left(D_{R}=\sqrt{K_{v}} D_{\text {scaled }}\right)$ and distances between channels vary also depending on thermalhydraulic feedback.

\subsection{Horizontal legs}

As it is explained in Section 2.3, flow regime transitions in horizontal volumes (cold and hot legs) become essential during PWR SBLOCA scenario, and a specific scaling criterion, (12), must be considered for reproducing equivalent behaviors and cooldowns in scaled transients. This criterion is based on the dependencies with Froude number and stratified liquid level that Taitel and Dukler (1976) Taitel et al. (1980) established for horizontal transitions. RELAP5mod3 flow regime maps are also based on classical maps performed by Taitel and Dukler, hence analogue transitions and mixture interactions should be expected in RELAP5 scaled calculations after applying the (12) relantionship.

On the other hand, some relevant special models are not preserved when (12) is applied. If both (12) and (11) are accomplished, velocities between scaled cells vary as follows

$$
v_{R}=K_{v}^{1 / 5} v_{\text {scaled }}
$$

Hence, special correlations like Ransom Trapp choking flow model, CCFL model, Abrupt area change model and User-specified form losses model are not preserved given that they depend on fluid velocities (see Sections 4.3.1.4.1, 4.3.1.4.4,4.3.1.4.6 and 4.3.1.4.7 of Martinez-Quiroga (2014)). Some of them, specially choked flow and CCFL model, can have significant impact on cooldown behavior for SBLOCA scenario with high vapour fluxes along the hot legs, thus distorsions should be expected for the comparison of equivalent scenarios at distinct scales.

\subsection{Environment heat losses}

RELAPmod3 does not show a scaling distortion when power to volume scaling criterion is applied to the 1D heat conduction model (Section 4.3.2 of Martinez-Quiroga (2014)).

$$
\begin{gathered}
\frac{\left(T_{m}^{n+1}-T_{m}\right) \bar{G}_{m}}{\Delta t}=\left(T_{m}-T_{m-1}\right) k_{l m} \delta_{l m}^{s}+\left(T_{m+1}-T_{m}\right) k_{r m} \delta_{r m}^{s}+P_{f} P(t)\left(Q_{l m} \delta_{l}^{v} m+Q_{r} m \delta_{r m}^{v}\right) \\
\bar{G}_{m}=\bar{\rho}_{l m} \delta_{l m}^{v}+\bar{\rho}_{r m} \delta_{r m}^{v}
\end{gathered}
$$

Hence, as (29) is not scale dependent, heat fluxes will be preserved and the energy that is lost to the environment will vary just depending on the surface area of the heat structures boundaries. In that sense, as the scaling factor of the cross sectional areas (13) (15) is smaller than $K_{v}$ factor of fluid volumes that are connected to boundaries of the heat structures, heat losses must decrease when RELAP5mod3 nodalizations are scaled-up.

It is also worth mentioning that wall heat transfer coefficients can vary in the scaling process as a result of changing the hydraulic diameter and the lenght (for natural convection in horizontal legs). Nevertheless, scaling distortions in the heat transfer coefficients are significantly smaller than scaling distortions that result from differences in surface-to-volume ratio. 


\subsection{Non condensable and boron transport equations}

For the non condensable transport continuity equation, (31), RELAP5 will present scaling distortions. First and second parenthesis of (31) depend on, respectively, the volume and the cross sectional area of the cell. Therefore, for horizontal volumes, the scaling factors are different and equation (31) is not preserved.

$$
\begin{aligned}
& V_{L}\left[\rho_{g, L}^{n} X_{n, L}^{n}\left(\tilde{\alpha}_{g, L}^{n+1}-\alpha_{g, L}^{n}\right)+\alpha_{g, L}^{n} X_{n, L}^{n}\left(\tilde{\rho}_{g, L}^{n+1}-\rho_{g, L}^{n}\right)+\alpha_{g, L}^{n} \rho_{n, L}^{n}\left(\tilde{X}_{g, L}^{n+1}-X_{g, L}^{n}\right)\right] \\
& +\left(\dot{\alpha}_{g, j+1}^{n} \dot{\rho}_{g, j+1}^{n} \dot{X}_{n, j+1}^{n} v_{g, j+1}^{n+1} A_{j+1}-\dot{\alpha}_{g, j}^{n} \dot{\rho}_{g, j}^{n} \dot{X}_{n, j}^{n} v_{g, j}^{n+1} A_{j}\right) \Delta t=0
\end{aligned}
$$

With respect to boron transport equations, the geometric parameters cannot be isolated in the upwind difference scheme (32), thus the equation cannot be properly scaled. For the Godunov model (33), scaling distortions are only expected for horizontal volumes given that the $\frac{A}{V}$ factor is not preserved.

$$
\begin{gathered}
V_{L}\left(\rho_{b, L}^{n+1}-\rho_{b, L}^{n}\right)+\left(\dot{\rho}_{j+1}^{n} v_{j+1}^{n+1} A_{j+1}-\dot{\rho}_{j}^{n} v_{j}^{n+1} A_{j}\right) \Delta t=0 \\
\rho_{b, L}^{n+1}=\rho_{b, L}^{n+1}+\frac{\Delta t}{V_{L}} A_{j} F_{j}^{n}-\frac{\Delta t}{V_{L}} A_{j+1} F_{j+1}^{n}
\end{gathered}
$$

\section{Power to Volume Scaling Tool}

PVST (Power to Volume Scaling Tool) is a software developed by the ANT at the UPC for generating scaled input decks following the Power to Volume Scaling criterion. Its main purpose is to provide an easy way for obtaining and comparing counterpart nodalizations at different scales. It makes possible to analyze the distorsions that must appear between the RELAP5mod3 simulations as a result of the scaling criterion.

The tool, programmed in C language for a DOS environment, is based on the Master Thesis of Garcia and Pla (1997), that developed a preliminary version for processing RELAP5mod3.2 input decks. The software has been improved including the processing of the new RELAP5mod3.3 cards, thus enabling the use of different versions of RELAP5. New features have been also added in order to facilitate the analysis of the different distortions reported on Section 3. These are:

- To scale the input decks keeping the surface-to-volume ratio mentioned in Section 3.4 for the passive heat structures. It allows generating scaled input decks that preserve the impact of the environment heat losses.

- To scale the input decks considering the Froude Number in selected components. It allows generating scaled input decks that preserve flow regime transitions in horizontal legs.

The running of the program is as follows: PVST asks to the analyst for the name of the input deck to be scaled, and for the $K_{v}$ factor to be applied (see Figure 3). Then, general components, as pipes, branches, single volumes, single junctions, multiple junctions and heat structures, and also specific components, as accumulators, separators, turbines, CANDU channels, jetmixers and eccmixers, will be scaled as a default in the "SCALED.TXT" output deck, modifying by a $K_{v}$ factor the areas, the volumes, and the mass flow cards, and by a $\sqrt{K_{v}}$ the diameter cards. In addition, several questions are automatically formulated in order to scale properly specific cards that require additional information. In that sense, PVST asks for the presence or not of pumps, particular time dependent volumes and junctions, control variables and tables that must be scaled (not all are required), and heat structures with parallel channels. Finally, PVST asks the user if the scaled input deck must preserve the impact of the environment heat losses and the Froude number in particular horizontal volumes and heat structures (see Figure 4). With all this information, the selected input deck is processed in a while loop that generates the "SCALED.TXT" output file, that will be a counterpart input deck of the previous one following the particular PtoV criterion specified by the analyst. 


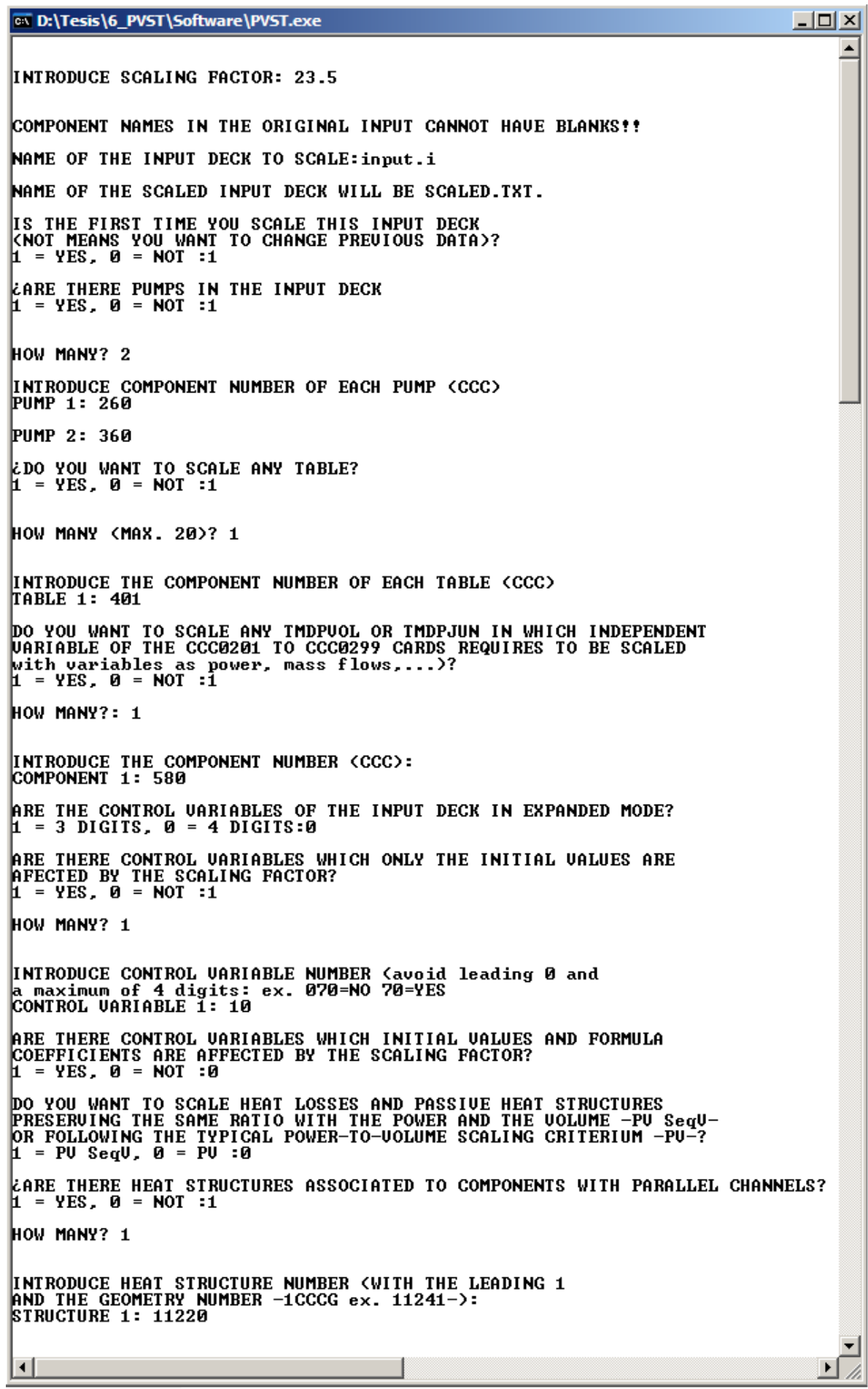

Figure 3: PVST general questions 


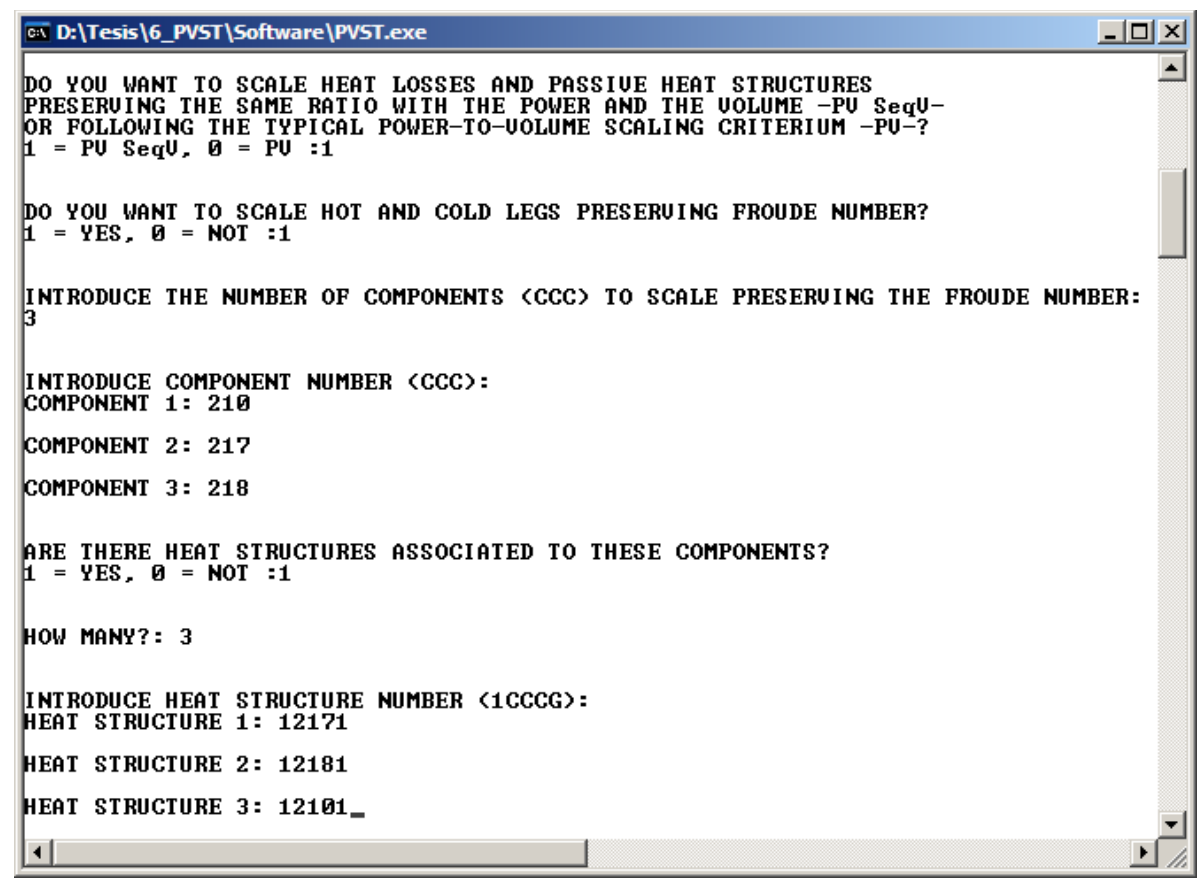

Figure 4: PVST particular scaling questions

Additionally, the code can also be run by the use of an input deck file where all the requests mentioned above are specified. This is helpful in order to generate several inputs at different scales or for doing sensitivity analyses of the effect of particular scaling distortions.

As it was mentioned previously, the aim of this software is not to give an answer of the PtoV scaling distortions when a $K_{v}$ factor is applied to a RELAP5mod3 input deck, rather the PVST package is a tool to ease the task of the analyst to generate a set of scaled input decks that will be used to evaluate the effect of the scale in the simulation results. In other words, by using the generated input decks at different scales, the analyst may be able to establish which of the scaling distortions reported in Section 3 modify the global response of a particular NPP scenario.

\section{Assessment of PVST software capabilities}

In order to show the capabilities and potentials of the PVST program, several scaled-up calculations have been performed over the original input decks of the OECD/NEA ROSA Test 3-1 and OECD/NEA PKL-2 Test G7.1. Test3-1 is introduced to show the main effects in the scaled-up simulations of the PtoV scaling factors ( Section 5.1) as well as the effect of the environment heat losses (Section 5.2) and the conservation of the hydraulic diameters in the DCs ( Section 5.3). The effect of the Froude number, which is important for flow transitions in horizontal pipes, could not be observed in that particular scenario. For this reason, in Section 5.4, which is devoted to show the effects of this phenomenon, another set of calculations is used regarding a different experiment (i.e. PKL-2 Test G7.1). The following is a description of the two experimental facilities and experiments used in the next subsections.

ROSA/LSTF is an experimental facility operated by JAEA, it is designed to simulate a Westinghousetype 4-loop 3,420 MWth PWR under accidental conditions, The ROSA-V Group (2003). It is a full-height and 1/48 volumetrically-scaled two-loop system with a maximum core power of $10 \mathrm{MW}$ (14\% of the scaled PWR nominal core power) and pressures scaled 1:1. Loops are sized to conserve volumetric factor $(2 / 48)$ and to simulate the same flow regime transitions in the horizontal legs (preserving $L / \sqrt{(} D)$ factor). Test 3-1, 
OECD/NEA (2007), simulates a SBLOCA (break size of $1 \%$ ) with scram failure and loss-of-offsite-power (HPI and LPI are unavailable). Due to the high-core power, supercritical natural circulation exists in the hot legs until the loops become empty. CCFL at the inlet of the steam generators and U-tubes during the two-phase natural circulation are objective of study in this test. A detailed description of this experiment as well as the main results obtained for the post-test simulation can be found in Martinez-Quiroga et al. (2012). For further details about the experiment and its simulation results, it is recommended to review the suggested report.

PKL is an ITF that reproduces the entire primary system and most of the secondary system (except for the turbine and condenser) of a 1300-MW PWR plant operated by AREVA Kremin et al. (2001). It is based on a 4-loop Siemens design (KWU), with elevations scaled 1:1 and volumes and power reduced by a factor of 145. PKL simulates all four loops separately. The operating pressure of the PKL facility is limited to 45 bars on the primary side and to 56 bars on the secondary side. This nevertheless allows simulation over a wide temperature range $(522 \mathrm{~K}$ to $322 \mathrm{~K})$, while requiring test procedure adaptations to address the high-pressure initial phase of typical PWR SBLOCA sequences. Test G7.1, Schoen and et al. (2012), is a counterpart experiment performed in PKL test facility as part of the OECD/NEA ROSA-2 and PKL-2 projects.The objective of both counterpart tests was mainly devoted to analyze the scaling effects between PKL and LSTF test facilities. The selected scenario was an upward oriented $1.5 \%$ hotleg SBLOCA with several system failures as no high-pressure safety injection and no automatic secondary-side safety cooldown. In Subsection part of the results of the LSTF-PKL Counterpart Test analyses performed in Martinez-Quiroga and Reventos (2014b), Freixa and et al. (2015) and Freixa and et al. (2016) are described in order to show the potentials of generating scaled-up input decks by using the option of preserving the Froude number.

Those volumetric and power parameters of the post-test calculations that are compared in the following figures have been multiplied by the $K_{v}$ factor in order to ease the comparison with the scaled-up calculations.

\subsection{PtoV scaled-up nodalizations}

A PtoV scaled-up nodalization (Scaled-up A) of the LSTF post-test 3-1 input deck was generated using PVST. This input deck was scaled to a comercial NPP size following the general criterion described by the relationship (6) (in particular, it was scaled to the Asco 2 NPP size (a Westinghouse PWR located in Spain) using a $K_{v}$ factor of 40 as suggested in Martinez-Quiroga et al. (2008)). Results of the main events (see table 1) showed a quite good agreement with the Post-test simulation until the entrance of the accumulators. Having a look into the details of the different thermal-hydraulic parameters of the transient, some discrepancies can be observed. The two most relevant discrepancies are:

- Secondary pressures: it can be seen that secondary pressure is higher in the scaled-up model (Figure 5): at the beginning of the transient, during natural circulation phase, secondary pressure increases faster in the scaled-up A nodalization and safety valves are opened (Figure 6); at the end of the transient, after the activation of the LSTF core protection system (that means, at very low power conditions, see Figure 7), secondary pressure of the Scaled-up A nodalization stabilizes at higher magnitudes.

- Core dryout: checking the values of Table 1 and the collapsed level of the core Figure 8, it can be noticed that losses through the break are lower for the scaled-up A nodalization (Figure 9), delaying the core dryout and the depressurization of the primary system.

Finally, it is also worth mentioning that Scaled-up A nodalization shows discrepancies in coolant temperatures of the secondary side. During the period of very low core power (after the initiation of the LSTF core-protection-system), liquid temperature in the riser is at saturation (see Figure 10). This phenomenon affects directly the collapsed liquid level of the riser, and therefore, the AFW closure condition (reported in the boundary conditions of Reference OECD/NEA (2007) -see also Table 1). As shown in Figure 10, temperatures of the Scaled-up A nodalization do not reach the saturation distorting the collapsed liquid level (Figure 11) and hence, advancing the closure of the AFW and the end of the depressurization action in the secondary side. 


\begin{tabular}{|c|c|c|c|}
\hline Event & Experimental[s] & Post-test 3-1[s] & Scaled-up A[s] \\
\hline Break & 0 & 0 & 0 \\
\hline $\begin{array}{l}\text { SCRAM signal: } \\
\text { Turbine trip and closure MSIV } \\
\text { PZR heater off } \\
\text { End of MFW and begin of AFW }\end{array}$ & 20 & 20 & 20 \\
\hline $\begin{array}{l}\text { Start of coastdown of primary } \\
\text { coolant pumps }\end{array}$ & 23 & 23 & 23 \\
\hline Primary coolant pumps stop & 272 & 272 & 272 \\
\hline $\begin{array}{l}\text { End of continuous opening of SG } \\
\text { RVs, End of two-phase natural cir- } \\
\text { culation, Break flow from one liquid } \\
\text { to 2-phase flow }\end{array}$ & About 300 & $300-400$ & $300-400$ \\
\hline $\begin{array}{l}\text { Core liquid level starts to decrease } \\
\text { (core uncovery) }\end{array}$ & About 1100 & 1150 & 1190 \\
\hline $\begin{array}{l}\text { Core power decrease by LSTF core } \\
\text { protection system }\end{array}$ & 1630 & 1725 & 1813 \\
\hline $\begin{array}{l}\text { Max. fuel rod surface temperature } \\
=930 \mathrm{~K}\end{array}$ & 1825 & 1891 & 1930 \\
\hline $\begin{array}{l}\text { Primary pressure lower than SG sec- } \\
\text { ondary pressure }\end{array}$ & About 1900 & About 1890 & About 1930 \\
\hline $\begin{array}{l}\text { Initiation of accumulator injection } \\
\text { system }(\mathrm{P}<4.5 \mathrm{MPa})\end{array}$ & About 2100 & 2180 & 2170 \\
\hline AFW stopped (SG level < $10.3 \mathrm{~m}$ ) & About 2700 & 2749 & 2539 \\
\hline $\begin{array}{l}\text { Loop seal clearing only in loop with- } \\
\text { out PZR }\end{array}$ & About 2200 & 2898 & 3140 \\
\hline End of the transient & 5547 & 5547 & 5547 \\
\hline
\end{tabular}

Table 1: Main events of OECD/NEA ROSA Test 3-1

\subsection{Assessment of the environment heat losses effect}

A new scaled-up input deck (Scaled-up B nodalization) was generated with PVST in order to analyze the effect of the environment heat losses. For this nodalization, the option of scaling-up the passive heat structures preserving the ratio between the heat exchange surface and the volume of coolant was activated (see Section 3.4). Figure 12 demonstrates that after activating this option, environment heat losses were preserved. In addition, Figures 13 and 14 demonstrate that by preversing the impact of the environment heat losses, primary-to-secondary heat transfer is reduced and secondary pressure trend is corrected (see Figure 5) obtaining a very similar behavior in both the post-test and the scaled-up B nodalization. Moreover, since system pressures are now more representative of the test conditions, break mass losses become similar (Figure 9) and the delay in the occurrence of the core dryout dissapears (Figure 8).

\subsection{Assessment of the hydraulic diameter effect}

In order to analyze the discrepancy reported in the coolant temperatures of the SGs, an additional Scaled-up C nodalization was generated using PVST. For that case, the hydraulic diameter of the SG DC was modified in order to obtain an idealized scaled-up input deck without scaling distortions effects. Hence, 


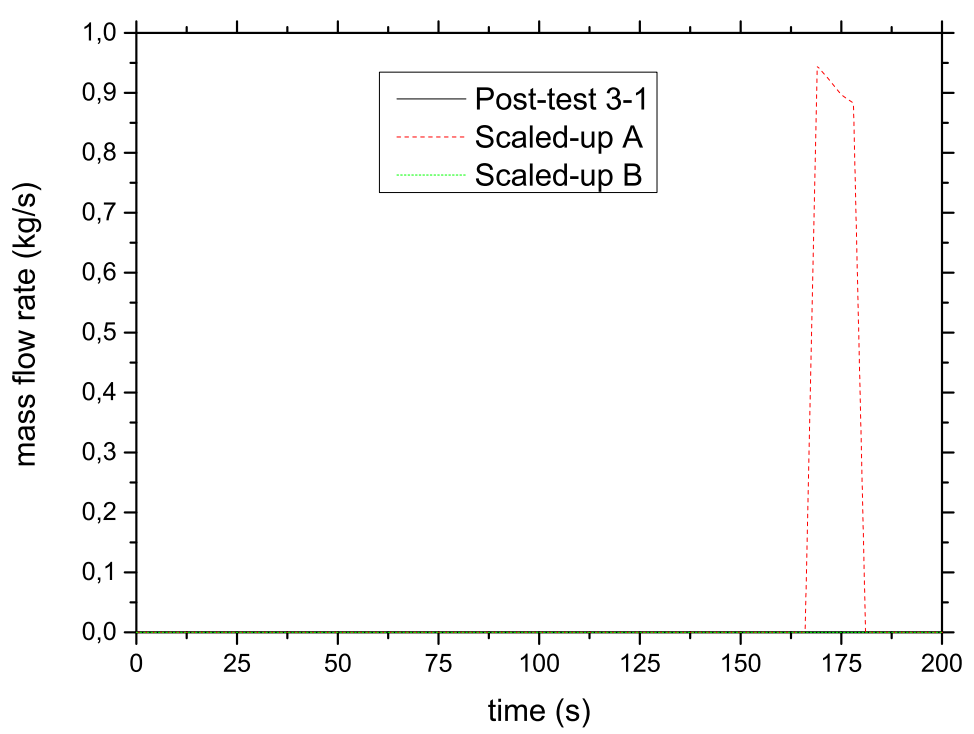

Figure 5: Secondary pressure

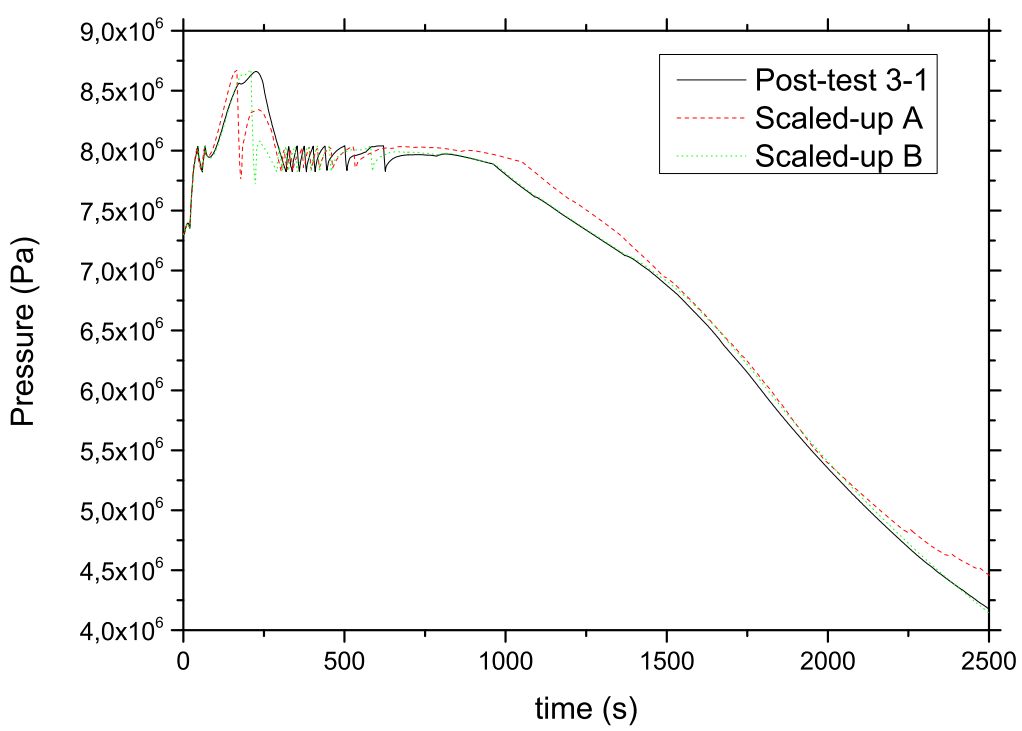

Figure 6: Safety valve mass flow rate

Scaled-up B nodalization was changed just preserving the hydraulic diameter of the post-test nodalization in the SGs DC. It was considered that this particular hydraulic diameter could have a relevant impact due to the following reasons: 


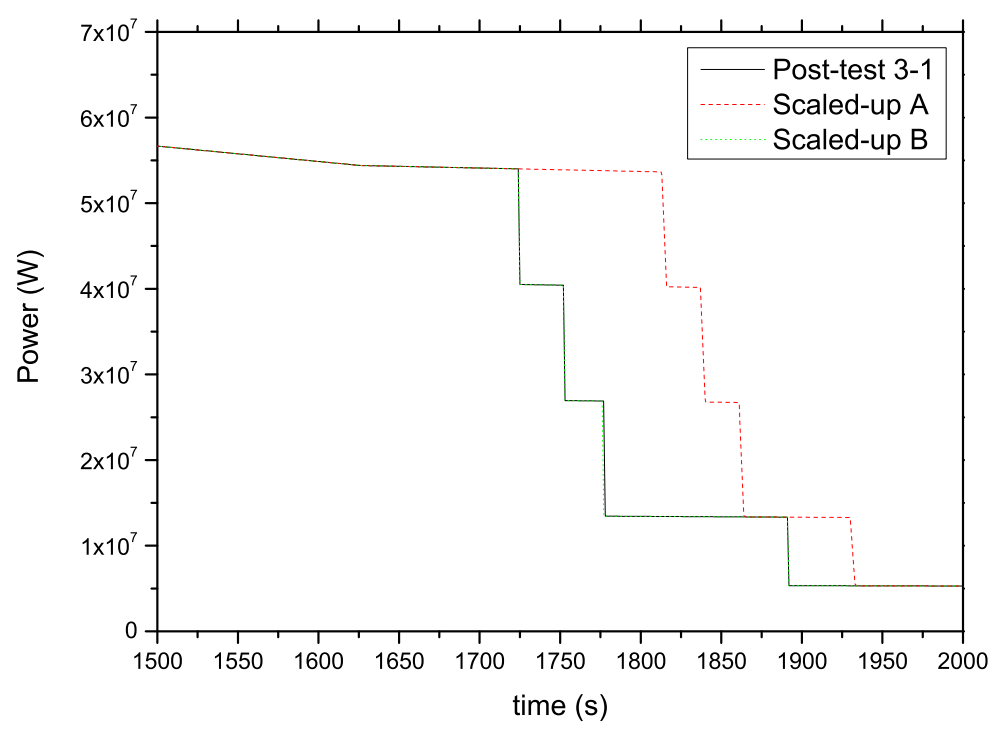

Figure 7: Core power

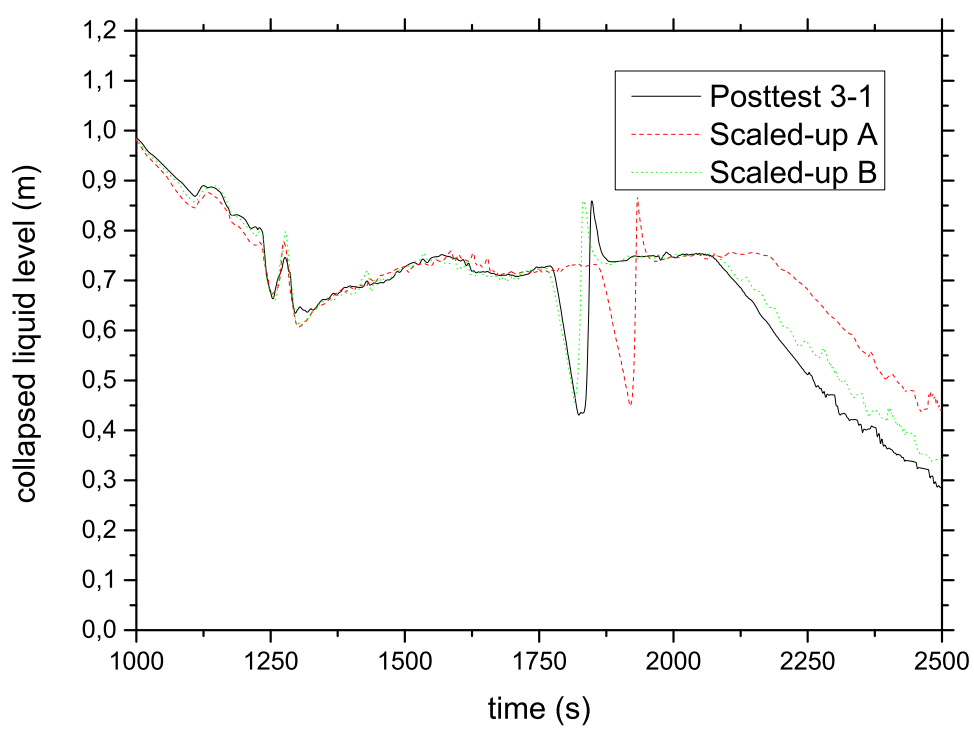

Figure 8: Core level

- After the activation of the LSTF core protection system, the secondary system can be considered to be isolated because the primary to secondary heat transfer drops drastically (see Figure 7). Under these conditions, effects of dragging, mixing, and interphase exchanges between the subcooled water 


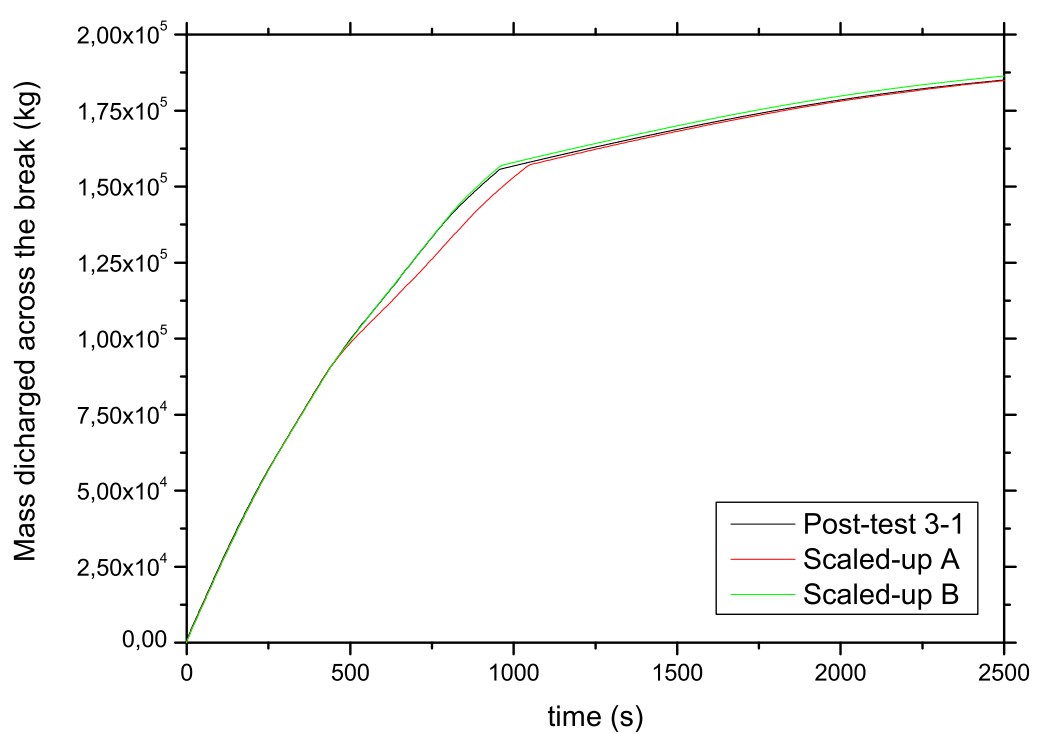

Figure 9: Total mass discharged across the break

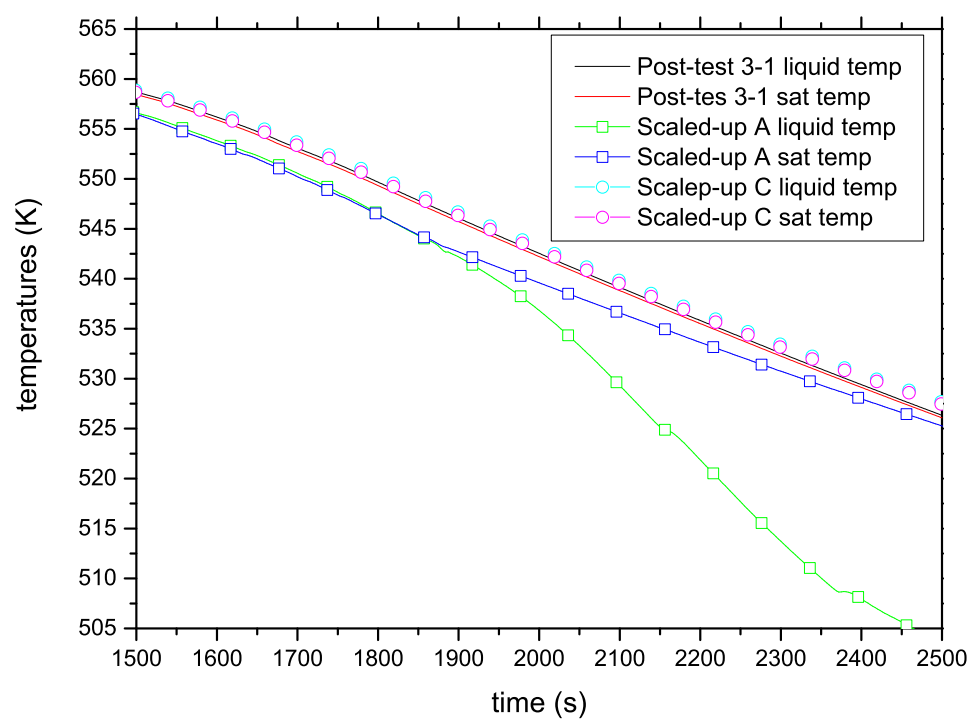

Figure 10: Liquid temperatures in the riser of the SG

of the AFW and the saturated vapour of the downcomer become dominant in the balance equations. All these effects are highly dependent on the hydraulic diameter of the SG DC. 


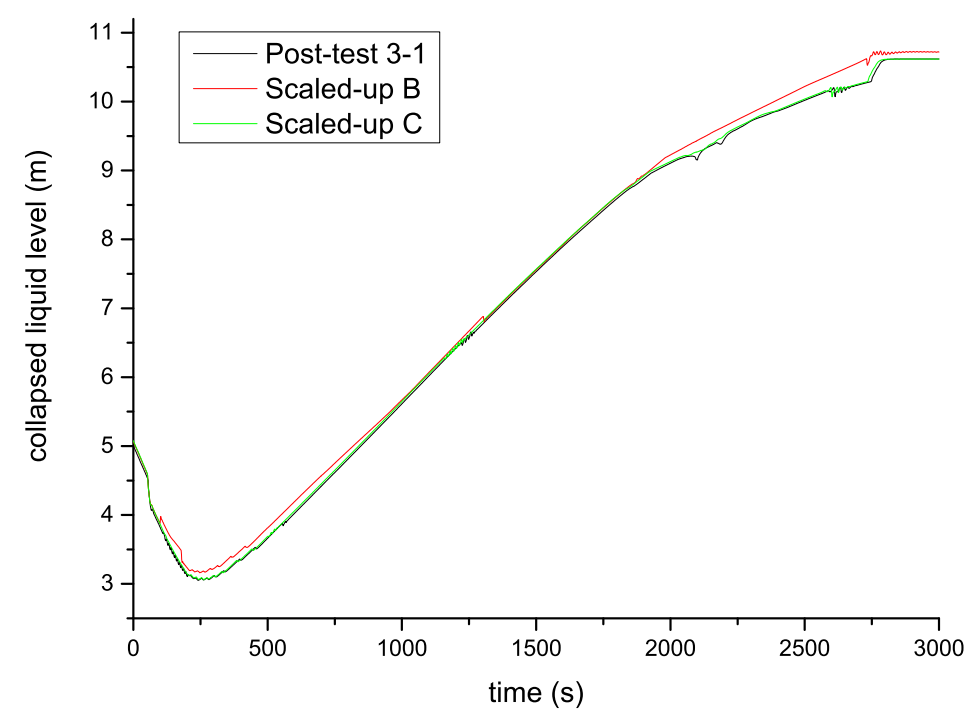

Figure 11: Collapsed liquid level in the riser of the SG

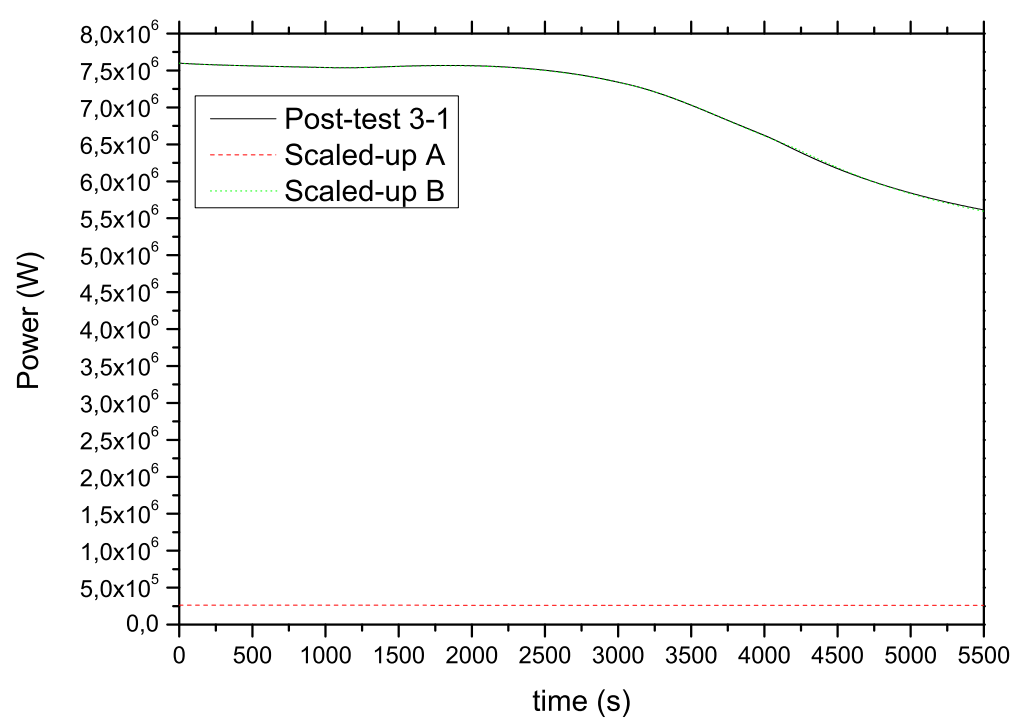

Figure 12: Environment heat losses

- Interphase interactions and drag correlations modelled by RELAP5mod3 closure equations are not preserved in scaled calculations when hydraulic diameter is modified.

The comparison between the Post-test calculation, the Scaled-up B nodalization, and the scaled-up C 


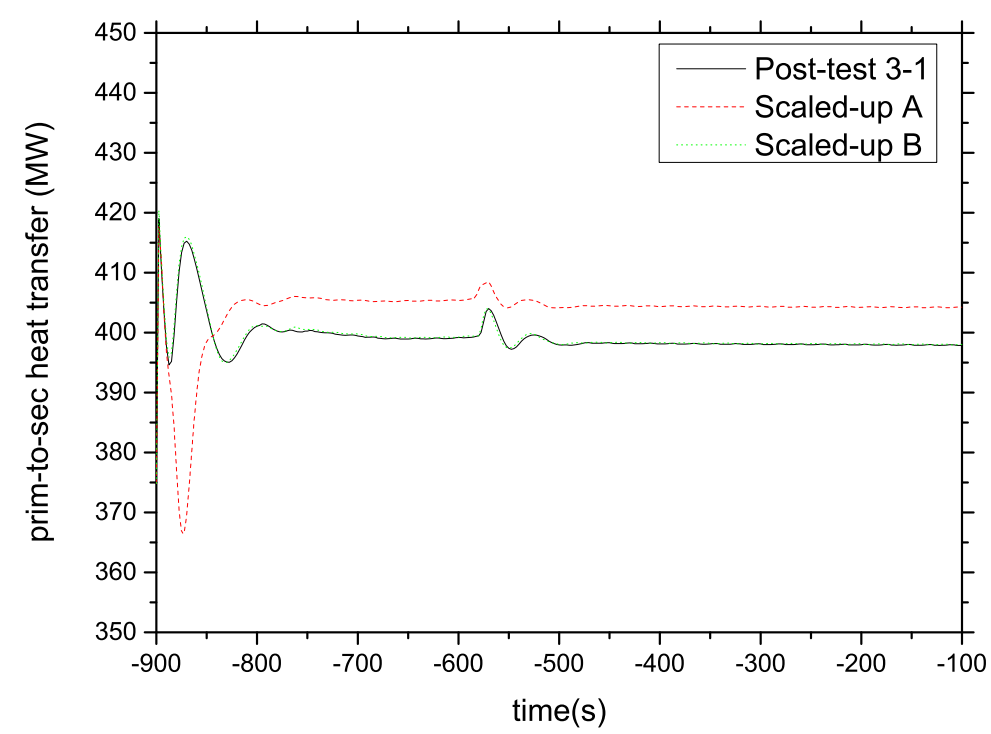

Figure 13: Primary-to-secondary heat transfer during conditioning phase

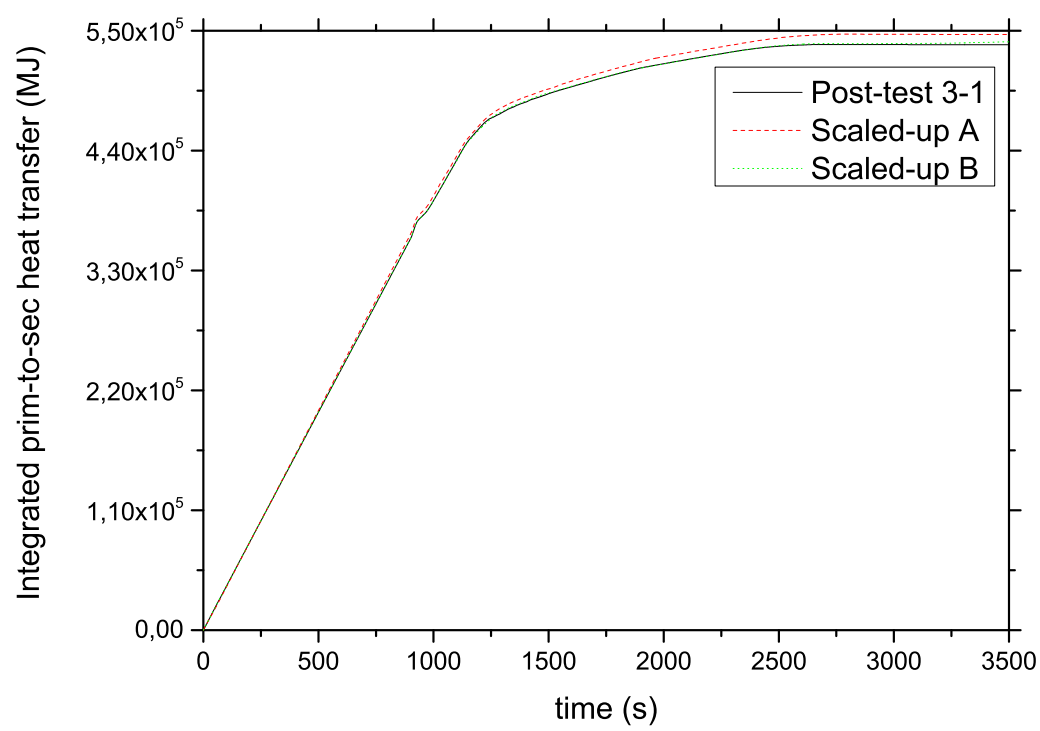

Figure 14: Energy transferred from primary to secondary during the transient

nodalization demonstrates that by preserving the hydraulic diameter in the DC the internal energies of the water in the DC are corrected (Figure 15), obtaining equivalent temperatures (Figure 10) and collapsed liquid levels (Figure 11) in the raiser of the SGs. 


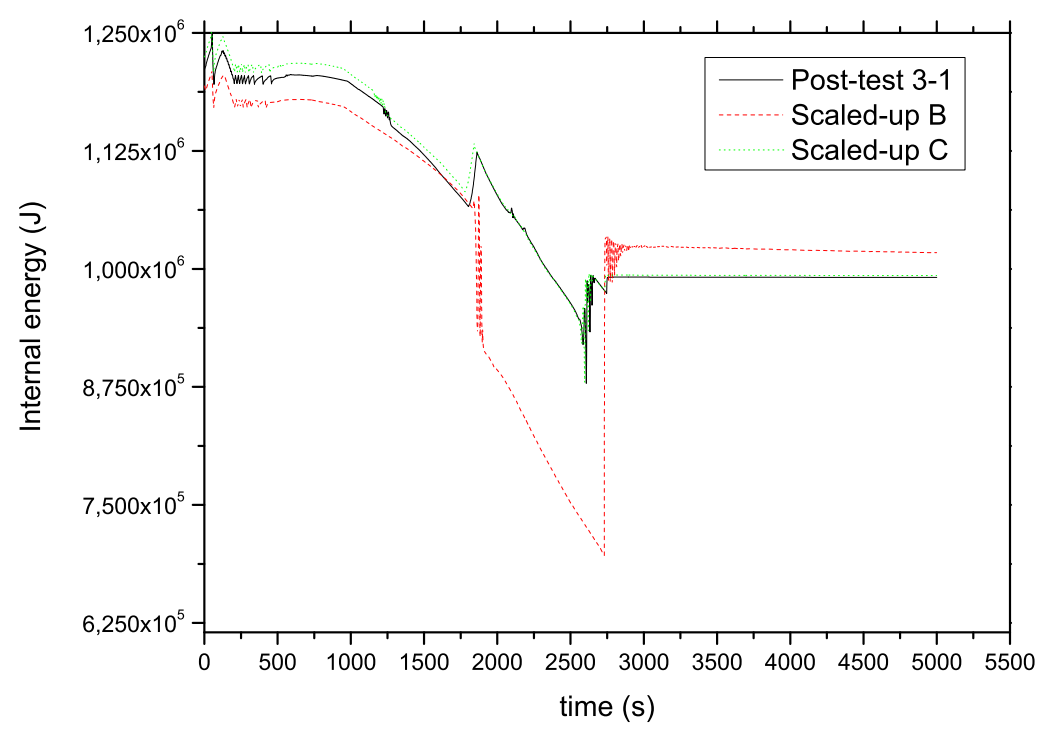

Figure 15: Internal energy of the liquid in the SG DC

\subsection{Assessment of the Froude number effect}

Several scaled-up calculations were carried out related to Post-test 3-1 in order to evaluate the impact of the Froude number without success. After correcting the effect of the environment heat losses, break mass flow rates became very similar during the two phase natural circulation and reflux and condenser phases (Figure 5). Therefore no relevant distortions were detected in the flow regime transitions before core dryout.

On the other hand, the effects of the Froude number conservation was observed in a different work published by authors Martinez-Quiroga et al. (2014c). In that work, several scaled-up calculations were performed for the PKL Test G7.1 in order to identify the effects of the environment heat losses and the Froude number in the scaling up of the experiment from PKL to LSTF. In particular, for the assessment of the Froude number effect, three scaled-up nodalizations were produced and compared with the results of the PKL counterpart post-test simulation:

- Sc-up nodalization A: a regular scaled-up nodalization.

- Sc-up nodalization B: a regular scaled-up nodalization preserving environment heat losses.

- Sc-up nodalization C: a regular scaled-up nodalization preserving environment heat losses and Froude number.

Comparison between the post-test, Sc-up nodalization B and Sc-up nodalization C revealed that the break mass flow discrepancies appeared as a result of a different Froude number (see Figure 16). The main discrepancies in core dry out and peak cladding temperature were removed once these changes were applied (see Figure 17).

\section{Conclusions}

The UPC has developed PVST, a system codes software that enables RELAP5mod3 input decks to be scaled by following the Power-to-Volume scaling criterion. This tool has revealed of great value to 


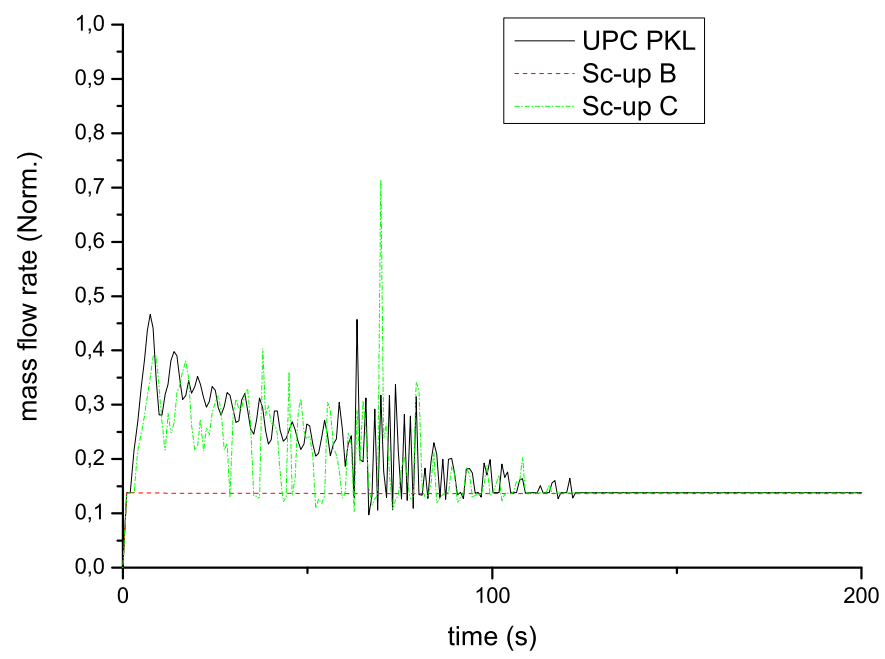

Figure 16: Break mass flow rate

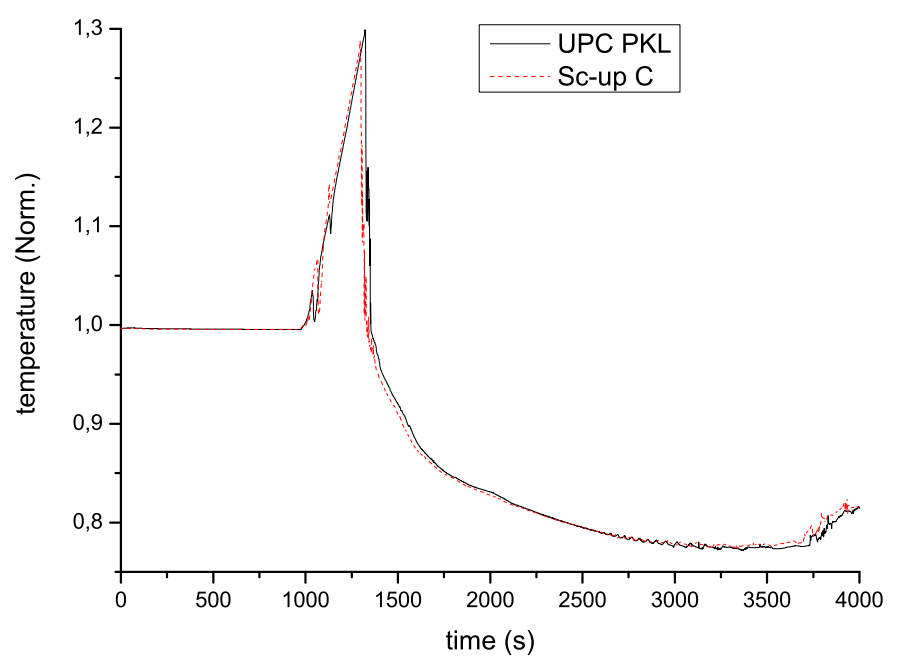

Figure 17: Core exit temperature

extrapolate ITF post-test simulations to NPP size by generating scaled-up nodalizations. In the present paper, the main features of the software have been introduced as well as the scaling distortions that can be expected in the scaled-up simulations as a result of the particular characteristics of the RELAP5mod3 equations and correlations.

With regards to code scaling distortions, several findings have been reported. Discrepancies are expected in fluid heat exchange with passive HSs as well as in flow regime transitions for the horizontal volumes (if $L / \sqrt{D}$ is not preserved) when a $K_{v}$ factor is applied to an ITF nodalization. Furthermore, if $L / \sqrt{D}$ is 
preserved, distortions will appear in the results of the Ransom \& Trapp choked flow model as well as in the CCFL correlation and in the form losses and local $\Delta$ Ps. If crossflows and transversal momentum equations are activated in multi-channel regions, distortions can also be expected under significant asymmetrical transient conditions. Furthermore, for vertical volumes, differences can be expected under mixture conditions as a result of the changes in the hydraulic diameters. Finally, results for the non-condensable transport continuity equation and the Godunov scheme will also vary with the application of the PtoV scaling criterion and the preservation of the $L / \sqrt{D}$ factor respectively.

It is worth mentioning that scaling distortions do not necesary mean significant effects in scaled-up calculations. Hence, additional features have been included in the software for assessing the effects of the hydraulic diameter, environment heat losses, and Froude number conservarion in both the overall and local behaviour of ITF experiments. Several calculations have been done on the qualified OECD/NEA ROSA Test 3-1 and OECD/NEA PKL-2 counterpart Test G7.1 simulations. Results have shown which phenomena modify the overall behaviour of the tests as well as the capabilities of the software to help the analyst to identify them. The remaining scaling distortions that could affect the final results of the calculations (changes in Ransom \& Trapp correlation results, CCFL model, form losses, and boron transport equations,...) have not been assessed because no significant differences have been noticed between the ITF calculations and the scaled-up calculations once the other scaling distortions were removed. Nevertheless, they could be easily tested with the same software for other transients in which they could be relevant.

\section{Acknowledgements}

This paper contains findings that were produced withinthe OECD/NEA ROSA2 and OECD/NEA PKL2 projects. The authors are grateful to the Management Board of bothprojects for their consent to this paper. The authors also want to thank the Spanish Safety Council that has partially funded this research.

\section{Bibliography}

\section{References}

Cabernier, W., Chudnik, R. A., 1969. Similitude Considerations for Modeling Nuclear Reactor Blowdowns (Trans. Am. Nucl. Sac., Vol. 12, 361).

Freixa, J., et al., 2015. Modelling guidelines for core exit temperature simulations with system codes (Nuclear Engineering and Design 286 116-129).

Freixa, J., et al., 2016. Qualification of a full plant nodalization for the prediction of the core exit temperature through a scaling methodology (Nuclear Engineering and Design 308 115-132).

Garcia, M. D., Pla, P., 1997. Diseño de una herramienta de ayuda para la preparacó en de modelos termohidráulicos a escala en centrales nucleares. Aplicación a dos experimentos. Master's thesis, Universitat Politècnica de Catalunya, in spanish.

Kremin, H., limprecht, H., Guneysu, R., Umminger, K., july 2001. Description of the PKL III Test Facility. Tech. rep., Framatome ANP report.

Martinez-Quiroga, V., 2014. Scaling-up methodology, a systematical procedure for qualifying NPP nodalizations. Application to the OECD/NEA ROSA-2 and PKL-2 Counterpart test. Ph.D. thesis, Universitat Politècnica de Catalunya.

Martinez-Quiroga, V., Reventos, F., 2014b. The Use of System Codes in Scaling Studies: Relevant Techniques for Qualifying NPP Nodalizations for Particular Scenarios (Science and Technology of Nuclear Installations, vol. 2014, Article ID 138745, 13 pages, 2014. doi:10.1155/2014/138745).

Martinez-Quiroga, V., Reventos, F., Freixa, J., 2014c. Applying UPC Scaling-Up Methodology to the LSTF-PKL Counterpart Test (Science and Technology of Nuclear Installations, vol. 2014, Article ID 292916, 18 pages, 2014. doi:10.1155/2014/292916).

Martinez-Quiroga, V., Reventos, F., Pretel, C., 2012. Post-Test Calculation of the ROSA/LSTF Test 3-1 Using RELAP5/Mod3.3. Tech. Rep. NUREG/IA-0409.

Martinez-Quiroga, V., Reventos, F., Pretel, C., Sol, I., 2008. Code Validation and Scaling of the ROSA/LSTF Test 3-1 experiment. No. International Topical Meeting on Safety of Nuclear Installations TopSafe 2008.

Navahandi, A. N., Castellana, S., Moradkhaniav, E., 1979. Scaling laws for modeling nuclear reactor systems (Nuclear Science and Engineering, 72).

OECD/NEA, 2007. Quick-look Report of ROSA/LSTF Test 3-1 (High Power Natural Circulation Experiment SB-CL-38 in JAEA). Tech. Rep. OECD/NEA ROSA project.

OECD/NEA, 2017. A state-of-the-art report on scaling in system thermal-hydraulics applications to nuclear reactor safety and design. Tech. Rep. NEA/CSNI/R(2016)14, Paris, France. 
Schoen, B., et al., 2012. PKL III G7.1: SB-LOCA with total failure of HPSI system (Counterpart Testing with ROSA/LSTF - Quick Look Report-. Tech. Rep. Internal report, AREVA.

Taitel, Y., Bornea, D., Dukler, A. E., 1980. Modeling Flow Pattern Transitions for Steady Upward Gas-Liquid Flow in Vertical Tubes (AIChE Journal. pp. 345-354).

Taitel, Y., Dukler, A. E., 1976. Model of Predicting Flow Regime Transitions in Horizontal and Near Horizontal Gas-Liquid Flow (AIChE Journal. 22. pp. 47-55).

The RELAP5 Code Development Team, 2001a. Relap5/Mod3.3 Code Manual. Volume I: Models And Correlations.

The RELAP5 Code Development Team, 2001b. Relap5/Mod3.3 Code Manual. Volume IV: Models And Correlations.

The ROSA-V Group, January 2003. ROSA-V Large Scale Test Facility (LSTF) System Description for the Third and the Fourth Simulated Fuel Assemblies. Tech. Rep. JAERI-Tech 2003-037, JAERI.

Ybarrondo, L., et al., 1974. Examination of LOFT Scaling. No. ASME Paper 74-WA/HT-53, ASME Annual Winter Meeting New York.

Zuber, N., 1980. Problems in Modeling of Small Break LOCA. Tech. Rep. NUREG-0724. 